\title{
EXPERIENCIAS MONÁSTICAS ENTRE LAS CORTES DE ESPAÑA Y SABOYA: EL CASO DE LOS EREMITAS CAMALDULENSES ENTORNO AL 1600.
}

\author{
Paolo Cozzo \\ (Università degli Studi di Torino) \\ paolo.cozzo@unito.it
}

\section{RESUMEN}

A finales del siglo XVI, la corte de Habsburgo favoreció el intento de implantar en España la experiencia eremítica camaldulense. Esta congregación monástica, nacida en Toscana en la Edad Media, fue introducida simultáneamente en Piamonte por iniciativa de la corte saboyana. El artículo trata de leer en paralelo las dinámicas diplomáticas y eclesiásticas de este proyecto que tuvo resultados diferentes en los dos contextos geopolíticos entonces vinculados por estrechos vínculos dinásticos.

PALABRAS CLAVE: Orden Camaldulense; eremitas; cortes; España; Saboya.

\section{MONASTIC EXPERIENCES BETWEEN THE COURTS OF SPAIN AND SAVOY: THE CASE OF CAMALDULENSIAN HERMITS AROUND 1600}

\begin{abstract}
At the end of the sixteenth century the Habsburg court favored the attempt to plant in Spain the Camaldulensian hermitic experience. This monastic congregation, born in Tuscany in the Middle Ages, was simultaneously introduced in Piedmont on the initiative of the Savoy court. The article tries to read in parallel the diplomatic and ecclesiastical dynamics of this project that had different results in both geopolitical contexts united by close dynastic ties.
\end{abstract}

KEY WORDS: Camaldulensian Order; hermit; courts, Spain; Savoy. 
En las últimas décadas, el clero regular ha merecido una especial atención por parte de la historiografía interesada en estudiar "religión, conflictividad y cultura" en la Europa de Antiguo Régimen ${ }^{1}$. Las investigaciones han permitido, en particular, conocer una faceta de ese clero: el papel que algunos religiosos, pertenecientes a las órdenes religiosas mendicantes (en concreto, dominicos, franciscanos y carmelitas) y a las congregaciones religiosas fundadas en el siglo XVI (fundamentalmente los jesuitas), desempeñaron en el marco de la corte ${ }^{2}$. Este interés por el "clero de corte" ha permitido descubrir como todos estos religiosos se valieron generalmente de la cercanía que disfrutaron con respecto al soberano - así como también a los altos dignatarios - para defender unas premisas político-ideológicas determinadas, gracias al desempeño de una serie de cargos eclesiásticos-clave dentro del organigrama cortesano: los de confesor real o director espiritual, predicador y capellán real. Así estos regulares supieron tejer, en torno a su persona o la de algún otro patrón cortesano, nutridas redes clientelares típicas de la "Iglesia en Palacio"3.

Con todo, no debemos olvidar que el clero de corte es un fenómeno bastante complejo. Su estudio y análisis no pueden ser abordados de manera homogénea y a gran escala porque son evidentes las especificidades locales y el condicionamiento por las coordenadas geográfico-espaciales de cada territorio. Unas especificidades que, naturalmente, requieren de una necesaria contextualización y de una adecuada profundización. Por poner sólo un ejemplo: las cortes italianas de los siglos XVI y XVII acogieron a un buen número de barnabitas, teatinos o filipenses ${ }^{4}$, a diferencia de

\footnotetext{
${ }^{1}$ Massimo Carlo Giannini, ed., Religione, conflittualità e cultura. Il clero regolare nell'Europa d'Antico Regime (Roma: Bulzoni, 2006).

${ }^{2}$ Flavio Rurale, ed., I religiosi a corte: teologia, politica e diplomazia in antico regime: atti del Seminario di studi: Georgetown University a Villa Le Balze, Fiesole, 20 ottobre 1995 (Roma: Bulzoni, 1998); Robert Bireley, The Jesuits and the Thirty Years War: Kings, Courts and Confessors (Cambridge: Cambridge University Press, 2003); Fernando Negredo del Cerro, Los predicatores de Felipe IV : corte, intrigas y religión en la España del Siglo de Oro (Madrid: Actas, 2006); José Martínez Millán, Manuel Rivero Rodríguez y Gijs Versteegen, eds., La Corte en Europa: Politica y Religión (siglos XVI-XVIII) (Madrid: Polifemo, 2012); Benoist Pierre, La monarchie ecclésiale: le clergé de cour en France à l'époque moderne (Seyssel: Champ Vallon, 2013); Paolo Cozzo, "Le clergé de cour entre service spirituel et fonction politique”, en L'État, la cour et la ville. Le duché de Savoie au temps de Christine de France (1619-1663), ed. Giuliano Ferretti (París: Classiques Garnier, 2017), 251-269; José Eloy Hortal Muñoz, Félix Labrador Arroyo, Jesús Bravo Lozano y África Espíldora García, eds., La configuración de la imagen de la Monarquía Católica. El ceremonial de la Capilla Real de Manuel Ribeiro (Madrid: Iberoamerica Vervuert, 2020).

${ }^{3}$ Rafael Valladares Ramírez, ed., La Iglesia en Palacio. Los eclesiásticos en las cortes hispánicas (siglos XVIXVII) (Roma: Viella, 2019).

${ }^{4}$ Véase, por ejemplo, el caso de la corte saboyana: Paolo Cozzo y Frédéric Meyer, "Deux fois, deux lois, une nation? Géographie ecclésiastique et vie religieuse, XVIe-XVIII siècles", en Les états de Savoie du duché à l'unité d'Italie, ed. Giuliano Ferretti (Paris: Classiques Garnier, 2019), 386-397. Más en general, sobre estas congregaciones véase: sobre los teatinos: Andrea Vanni, "Fare diligente inquisitione": Gian Pietro Carafa e le origini dei Chierici regolari teatini (Roma: Viella, 2010); Domenico Antonio D'Alessandro, ed., Sant'Andrea Avellino e i teatini nella Napoli del viceregno spagnolo. Arte, religione, società (Nápoles: D’Auria, 2011); sobre los barnabitas: Elena Bonora, I conflitti della controriforma. Santità e obbedienza nell'esperienza religiosa dei primi barnabiti (Florencia: Le Lettere, 1998); sobre los filipenses: Franco Bolgiani, Gian Franco Gauna, Antonio Gobbo y Giuseppe Goi, eds, Oratorio e laboratorio: l'intuizione di san Filippo Neri e la figura di Sebastiano Valfré (Bolonia: Il Mulino, 2008).
} 
los territorios no italianos donde, si bien se documenta la presencia de dichos regulares, no con la misma intensidad y frecuencia. Por lo demás, Italia es el espacio geopolítico en el que nacieron las congregaciones de "chierici regolari" y en el que, además, maduraron sus prácticas y experiencias de vida religiosa, que se expresaron también en los contextos cortesanos.

En cambio, las familias religiosas monacales han recibido una menor atención, quizás debida a una aparentemente menor difusión e implantación de sus miembros por las cortes europeas. En España, por ejemplo, en Antiguo Régimen, "solo religiosos de cinco Ordenes - y básicamente tres" (dominicos, franciscanos y jesuitas) - "se ocuparán del confesionario real" . Pero lo cierto es que el clero de corte contó también con significativas figuras dentro de las ordenes monacales, como es confirmado por el papel - entre otros - del jerónimo Hernando de Talavera (1428-1507), confesor de la reina Isabel de Castilla. Al respecto de la presencia monástica en la corte, se mencionan los estudios sobre los cistercienses reformados del monasterio francés de Feuillant. Se trata de una reforma religiosa, de carácter rigorista, desarrollada en la Francia de la segunda mitad del siglo XVI - en particular, en el monasterio cerca de Tolosa del que los monjes recibieron el nombre de Feuillants -, cuyos miembros dejaron sentir su influencia en la corte de París entre el Quinientos y el Seiscientos ${ }^{6}$. Un tanto parecido sucede con Italia, donde estos monjes tuvieron una notable presencia, especialmente en el ducado de Saboya ${ }^{7}$. Por otro lado, recientes trabajos publicados sobre el cenobitismo y el eremitismo camaldulenses han puesto de relieve el papel desempeñado, en calidad de directores espirituales y consejeros, por interesantes figuras camalduenses, es decir hijos de la familia monástica de Camaldoli.

\section{CAMALDOLI, UN “CASTILLO DE LA ORACIÓN”}

Camaldoli es una experiencia benedictina que, sobreviviendo al carisma de su fundador (san Romualdo, vivido entre los siglos X y XI), se institucionalizó y logró consolidarse en el tiempo y en el espacio ${ }^{8}$. Erigiéndose como uno de los más importantes "castillos de la oración" del Occidente latino", Camaldoli se convirtió en centro propulsor de una irradiación monástica que, durante la Edad Media y la primera Edad Moderna, supo involucrar primero a la península italiana, y luego a toda Europa.

En la Edad Moderna se multiplicaron los intentos de reforma rigorista, que acentuaron la prevalencia del componente eremítico sobre el cenobítico. Esto implicó

\footnotetext{
${ }^{5}$ Leandro Martínez Peñas, El confesor del Rey en Antiguo Régimen (Madrid: Editorial Complutense, 2007), 790-791.

${ }^{6}$ Benoist Pierre, La bure et le sceptre: la congregation des Feuillants dans l'affirmation del États et des pouvoirs princiers (vers 1560-vers 1660) (Paris: Publications de la Sorbonne, 2006).

${ }^{7}$ Gianfranco Armando, Silvia Beltramo, Paolo Cozzo y Cristina Cuneo, eds., I cistercensi foglianti in Piemonte fra corte e chiostro (secoli XVI-XIX) (Roma: Viella, 2020).

${ }^{8}$ Cecile Caby y Pierluigi Licciardello, eds., Camaldoli e l'Ordine camaldolese dalle origini alla fine del XV secolo: Atti del I Convegno internazionale di Studi in occasione del millenario di Camaldoli (1012-2012), Monastero di Camaldoli, 31 maggio-2 giugno 2012 (Cesena: Centro storico benedettino italiano, 2014).

${ }_{9}$ Glauco Maria Cantarella, ed., I castelli della preghiera. Il monachesimo nel pieno medioevo (secoli X-XII) (Roma: Carocci, 2020).
} 
tensiones y fisuras dentro de la Orden, donde comenzaron a formarse ramas autónomas (como la congregación de Monte Corona) cuyas relaciones con Camaldoli a menudo fueron conflictivas. Más tarde, los repetidos intentos de recomposición tuvieron que enfrentarse al nacimiento, en el ducado de Saboya y en Francia durante el siglo XVII, de nuevas congregaciones definidas sobre una base territorial.

Hay que comprender este proceso a la luz de la tendencia - típica de la primera Edad Moderna - a la nacionalización de las órdenes religiosas. Camaldoli, que los Medici (y, después ellos, los Lorena) siempre habían protegido y favorecido ${ }^{10}$, de hecho, era percibida como una fundación monástica toscana, mientras que las de Piamonte ${ }^{11}$ y de Francia ${ }^{12}$ se veían afectadas por las interferencias políticas y diplomáticas impuestas por las cortes de Turín y de París. A pesar de otros intentos de expansión, a finales del siglo XVII la Orden camaldulense estaba dividida en cuatro congregaciones: las de Camaldoli, Monte Corona, Piamonte y Francia.

En los distintos contextos políticos donde se establecieron, los camaldulenses supieron tejer relaciones con soberanos y cortes. Muy emblemática, en ese sentido, es la figura del padre Alessandro Ceva. Este monje pertenecía a una antigua familia de la nobleza del Piamonte que había estado, durante mucho tiempo, al servicio de los Saboya. Tras haber ocupado el generalato de la congregación camaldulense (cuya casa madre, Camaldoli, estaba ubicada en Toscana), en 1596 abandonaba el Granducado mediceo y regresaba al ducado saboyano donde decidía fundar una congregación camaldulense autónoma: la de Piamonte. El haber Alessandro Ceva asistido espiritualmente a los duques de Saboya, Carlos Manuel I y Catalina Micaela, ayudó a este propósito, de lo cual se deduce que los camaldulenses contaron siempre con el favor de la corte turinesa. Ceva es, en definitiva, el responsable de la difusión de los camalduleses por los territorios ducales: así, bajo el patrocinio de los príncipes saboyanos, esta congregación logró fundar cuatro ermitas ${ }^{13}$.

Por lo que concierne a España, la experiencia camaldulense no terminó de arraigar, a pesar de que era conocida gracias a los viajes que algunos monjes hispanos habían hecho a Italia en el siglo XV. Es el caso de un beato, de nombre Eugenio, que, durante la segunda mitad del Quattrocento, fue en el monasterio veneciano de San Miguel. Se menciona también a un monje catalán, el beato Pellegrino Palau de Barcelona

${ }^{10}$ Gaetano Greco, "Firenze e i camaldolesi dai Medici ai Lorena", en L'Ordine camaldolese in età moderna e contemporánea, secoli XVI-XX (Atti del II Convegno di studi in occasione del millenario di Camaldoli 10122012), eds. Giuseppe Maria Croce y Ugo A. Fossa (Cesena: Badia di Santa Maria del Monte, 2015), 17 70.

11 Gianfranco Armando, Laura Facchin y Diego Lanzardo, eds., Gli eremiti camaldolesi di Piemonte: 1601-1801, volume di studi derivato dal convegno svoltosi nel Palazzo comunale di Cherasco il 14 novembre 2015 (Cherasco: Cherasco Cultura, 2017); Paolo Cozzo, “La presenza camaldolese in Piemonte", en L'Ordine camaldolese, 207-231.

12 Giuseppe Maria Croce, “«Mystique et rebellion»: la Congregazione francese degli eremiti camaldolesi”, en L'Ordine camaldolese, 233-346.

${ }^{13}$ Las ermitas fundadas en Piemonte fueran las de Pecetto (Turín), Cherasco, Busca y Lanzo. Paolo Cozzo, Un eremita alla corte dei Savoia. Alessandro Ceva e la nascita della Congregazione Camaldolese di Piemonte (Milán: Franco Angeli, 2018). 
(†1620), que alrededor de 1580 se encontraba ya residiendo en Camaldoli, donde vivió "encerrado" (es decir en la soledad más radical) durante cuarenta años ${ }^{14}$.

Así pues, nos ha parecido interesante reflexionar sobre los esfuerzos (por otra parte, fracasados) de expansión de los camaldulenses por tierras ibéricas, teniendo en cuenta no sólo la compleja relación entre la corte de los Habsburgo y la tradición monástica, sino también las implicaciones (políticas y diplomáticas) producidas por la introducción de otra experiencia regular.

\section{CAMALDULENSES EN ESPAÑA}

Un dato a tener en cuenta - y del que nos parece conveniente partir - es el hecho de que la fundación de la congregación camaldulense del Piamonte, que tuvo lugar entre los años 1596 (fecha de la llegada de Alessandro Ceva a Turín) y 1602 (fecha en que fue levantada la primera ermita, ubicada en una colina de la capital piamontesa), coincidió en el tiempo con los intentos de introducción de esta familia religiosa en la península ibérica. En efecto, es una época (marcada a nivel político y diplomático por la Paz de Vervins) en la que las relaciones entre las cortes de Madrid y Turín, pese al temprano fallecimiento de la infanta española Catalina Micaela en $1597^{15}$, continuaron siendo intensas, como viene a demostrarlo el viaje a España de los príncipes saboyanos bajo la supervisión de Giovanni Botero ${ }^{16}$.

Las primeras noticias sobre la presencia camaldulense en la península ibérica se remontan al año 1596, y están vinculadas a tres ermitaños hispanos (los frailes Gil de Granada, Rodolfo de Valencia y Diego), afiliados a la congregación camaldulense de Monte Corona (donde Gil, siendo sacerdote, era maestro de novicios, Rodolfo clérigo y Diego subdiácono) que regresaron del Piamonte con la intención de fundar un cenobio camaldulense en territorio hispano ${ }^{17}$. La iniciativa, aprobada por el papa Clemente VIII, fue presentada al rey Felipe II, cuya autorización era indispensable. El monarca, venciendo las resistencias del Consejo Real, se mostró disponible, pero impuso algunas condiciones cuya aceptación resultó harto complicado para la curia romana. La primera era que los camaldulenses (que tenían ya un asentamiento, ubicado al noroeste de la ciudad de Madrid, entre los actuales distritos de El Pardo y Fuencarral) únicamente podían acoger entre sus filas a religiosos de origen hispano. La segunda consistía en que debían estar sometidos, en exclusiva, a la jurisdicción del ordinario

${ }^{14}$ Ugo A. Fossa, Monaci a Camaldoli. Memorie, percorsi, interpretazioni (Camaldoli: Edizioni Camaldoli, 2020), 135; Ugo Dovere, "«Occuparsi di Dio solo». Temi e figure di spiritualità camaldolese tra Cinquecento e Settecento”, en L'Ordine camaldolese, 443.

${ }^{15}$ Sobre Catalina Micaela y su experiencia de duquesa de Saboya entre 1585 y 1597 véase Blythe A. Raviola y Franca Varallo, eds., L'Infanta. Caterina d'Austria, duchessa di Savoia (1567-1597) (Roma: Carocci, 2013).

16 María José Del Rio Barredo, "El viaje de los príncipes de Saboya a la Corte de Felipe III (16031606)", en L'affermarsi della corte sabauda. Dinastie, poteri, élites in Piemonte e Savoia fra tardo medioevo e prima età moderna, eds. Paola Bianchi y Luisa C. Gentile (Turín: Zamorani, 2006), 407-434; Blythe A. Raviola, Giovanni Botero. Un profilo fra storia e storiografia (Milán: Bruno Mondadori, 2020), 105-126.

${ }^{17}$ Ernesto Zaragoza Pascual, "Primera fundación de la Orden Camaldulense en España (15971602)", Studia monastica 28/2 (1986): 359; Croce, "Mystique et rebellion", 247. 
diocesano, y no a la del general de los camaldulenses ${ }^{18}$.

Como era natural, estas condiciones (sobre todo, la referida a la admisión de nuevos miembros) eran inaceptables para Roma. Desde la curia romana, se procedió a reafirmar las competencias del nuncio apostólico de Madrid en contra de las pretensiones del Consejo Real. Estas negociaciones, que comprometieron ambas cortes entre 1598 y 1600, retrasaron el proyecto de fundación de nuevos cenobios camaldulenses en la península ibérica. Esta situación se agravó, además, con el fallecimiento con fama de santidad de Gil de Granada (el fraile camaldulense español que, desde el principio, había defendido la iniciativa de fundar nuevos cenobios de su familia religiosa), así como por el cambio que tuvo lugar en el trono de la Monarquía hispana tras el óbito de Felipe II, en septiembre de 1598. Felipe III, quién volvió a retomar el proyecto de nuevas fundaciones camaldulenses en la península ibérica, hubo de aceptar las condiciones impuestas por el papa Clemente VIII y, asimismo, solicitar el envío, desde Italia, de religiosos camaldulenses.

En este contexto, fraile Rodolfo de Valencia (uno de los miembros de ese grupo inicial camaldulense hispano) se dirigía al duque de Sessa, embajador de Felipe III en la corte papal, en los términos siguientes: "Es cosa maravillosa ver la gran devoción que hay en este santo hábito, con la que muchos señores, sabiendo que yo había de fundar, me han favorescido" ${ }^{19}$. Que en la Monarquía hispana existía un cierto interés por esta nueva familia religiosa, parecen atestiguarlo algunos indicios. Uno de los más significativos es un cuadro, Alegoría de la Orden de los Camaldulenses, atribuido al Greco (o a su escuela pictórica), en el que a los pies de una ermita se encuentran representados los santos Benedicto y Romualdo. Bajo estos, aparecen las armas de dos importantes familias de la nobleza española (los Arcos y Añover y los Mendoza) ${ }^{20}$. Se cree que esta obra (hoy conservada en el museo del Instituto de Valencia de don Juan, pero originalmente custodiada en el castillo de Batres, donde estaba inventariada con el título "Retrato del monasterio de la Gran Camaldula") puede haber sido encargada por don Pedro Lazo de la Vega (1559-1637), primer conde de los $A r \cos ^{22}$, a quien se atribuye la intención (no realizada) de fundar un asentamiento camaldulense cerca de la nativa Toledo ${ }^{23}$. Este cuadro (que en efecto deja traslucir en el fondo un panorama que evoca la famosa Vista de Toledo del Greco) tal vez fue presentado por el conde de los Arcos al proprio Felipe III (del que había sido nombrado mayordomo mayor), para

18 Zaragoza Pascual, "Primera fundación”, 361-363.

19 Zaragoza Pascual, "Primera fundación”, 380.

${ }^{20}$ Ibídem, 361.

${ }^{21}$ Balbina Caviró Martínez, "Los Grecos de don Pedro Laso de la Vega”, Goya. Revista de arte 184 (1985): 216-226.

22 Santiago Martínez Hernández, "Discreto, artífice y erudito: Un retrato abocetado de don Pedro Laso de la Vega, conde de los Arcos, mayordomo de la reina Margarita de Austria y de Felipe IV (15591637)", en Las relaciones discretas entre las Monarquias Hispana y Portuguesa: Las Casas de las Reinas (Siglos XVXIX), eds. José Martínez Millán y María Paula Marçal Lourenço (Madrid: Polifemo, 2008), vol. II, 1187 1220.

${ }^{23}$ Oswaldo Chinchilla, "Pinturas emblemáticas y fiestas teatrales en la epoca colonial”, Conferencias del Museo Pupul Vuh (Museo Pupul Vuh, 2007) https://popolvuh.ufm.edu/wp-content/uploads/2019 $207 /$ Chinchilla2006.pdf, 7. 
defender la causa de la introducción de los camaldulenses en España, precisamente a partir de su ciudad natal. La notoriedad de los camaldulenses se vio alimentada también gracias a la redacción de una biografía edificante de San Romualdo, dada a la imprenta en Madrid en $1597^{24}$ por parte de Juan de Castañiza (1555-1599), monje, predicador, hagiógrafo y cronista perteneciente a la congregación benedictina de San Benito de Valladolid ${ }^{25}$.

Esta obra, cuyo autor dedicó a Felipe II con la intención de lograr su apoyo para una "petición tan justa de los padres camaldulenses, que han venido a esta corte a suplicar a Vuestra Majestad les dé licencia para introduzir en España religión tan santa" 26 , dio a conocer posiblemente -aunque, por otro lado, este aspecto resulta difícil de estimar y cuantificar- la experiencia romualdina entre las élites de la Monarquía hispana. Lo cierto es que, tras el abandono del proyecto inicial consistente en levantar un cenobio camaldulense en las cercanías de Madrid (al abrigo de la corte), tal vez motivado por las persistentes resistencias del Consejo Real, y el fracaso del proyecto toledano del conde de Arcos (del que todavía no tenemos informaciones suficientes), surgió otro nuevo que propuso trasladar la instalación camaldulense a Sevilla.

En la ciudad hispalense, Baltasar Brum y Silveira, un sacerdote portugués (nativo de la isla de Faial, en las Azores, pero de origines flamencos) afincado en la misma desde 1580, ponía a disposición de los camaldulenses, en noviembre de 1600, una de sus propiedades (quizás heredadas del humanista Benito Arias Montano, del cual fue albacea testamentario y heredero de unos bienes inmuebles ${ }^{27}$ ) ubicada en un lugar extramuros de la ciudad (conocido como Cantalobos), debido a la "particular devoción" que sentía por el carisma espiritual camaldulense ${ }^{28}$.

${ }^{24}$ Juan de Castañiza, Historia de S. Romualdo padre y fundador de la orden Camaldulense, que es vna idea y forma perfecta de la vida solitaria perfecta (Madrid: por el licenciado Castro, 1597). La traducción al italiano de esa obra: Historia della vita di s. Romualdo. Padre, e fondatore dell'ordine camaldolese, ch'è una idea, e forma perfetta della vita solitaria. Composta dal r.p.d. Gio. da Castagnizza ... Trasportata dalla lingua spagnola nella italiana da d. Timotheo da Bagno (Venecia: por Domenico Imberti, 1605), fue reeditada en Florencia en 1671 y en Nápoles en 1685 (Dovere, "Occuparsi di Dio solo", 439); una traducción francesa fue publicada en Lyon en 1616.

25 Ernesto Zaragoza Pascual, "Juan de Castañiza", Diccionario biográfico español, en línea: http://dbe.rah.es/biografias/26804/juan-de-castaniza (ultima consulta: 20/09/2021).

${ }^{26}$ Castañiza, Historia de S. Romualdo padre y fundador de la orden Camaldulense, 13.

${ }^{27}$ José María Miura Andrades, "Baltasar de Brum, albacea testamentario de Arias Montano y heredero de su finca en Campo de Flores", en Anatomía del humanismo. Benito Aria Montano, 1598-1998, Homenaje al profesor Melquiades Andrés, ed. Luis Gómez Canseco (Huelva: Universidad de Huelva, 1998), 455-475.

28 Zaragoza Pascual, "Primera fundación”, 382. Brum había donado "una grande quantità di terre con molti arbori de olive, de narangi, cedri, limoni et altri frutali, et con i cipressi, pino, palme et vigne et una caseria in mezzo del cerraglio che è molto grande con muri alti e forti, et in esso ci è un stanco assai grande con altre acque da piede, molino de oglio et furno con molte altre commodità, tutto il quale è stimato valuta di 50.000 scudi" (Ibídem, carta del fr. Rodolfo de Velencia, 27 de noviembre de 1600). Sobre la dimensión ecológica de la tradición camaldulense véase Paolo Cozzo, "Bosques y patrimonio forestal en la cultura monástica entre la Edad Media y Edad Moderna: el caso de la Congregación camaldulense", Manuscits (en prensa). 
Así pues, a través del duque de Sessa se pedía a Roma el envío de varios monjes camaldulenses italianos. En 1601 llegaban a Sevilla seis religiosos: tres procedentes de Camaldoli (los padres Tommaso Tomasi de Siena, Giovanni Battista Maiolini de Milán, Antonio Bonventuri de Florencia) y tres de la recién creada congregación del Piamonte ${ }^{29}$. Este último dato no parece en nada casual. Permite reconocer (tratándose todavía de una mera hipótesis por confirmar con otras investigaciones) el papel que pudo jugar la diplomacia saboyana en este campo, que vio, tal vez, la ocasión perfecta para asentar, de una vez por todas, en territorio hispano a la familia religiosa camaldulense, a la que ya consideraba una congregación "nacional".

\section{CAMALDOLI, LOS SABOYA Y LOS HABSBURGO: PARALELISMOS Y DIVERGENCIAS}

No hay que olvidar tampoco que la fundación de la congregación del Piamonte, nacida gracias al impulso de Alessandro Ceva y con el apoyo del duque Carlos Manuel I, había sido obstaculizada por Camaldoli ${ }^{30}$, oposición detrás de la cual se vislumbraba la sombra de los Medici, protectores tradicionales de la congregación eremítica cuya casa madre estaba ubicada en el granducado de Toscana. Si ya el regreso de Ceva al Piamonte, después de unos años difíciles al frente del generalato, ha de ser considerado como el resultado de un durísimo enfrentamiento entre los propios camaldulenses ( $\mathrm{y}$ también entre la facción "toscana", sostenida por la corte de Florencia, y las de las otras "naciones", deseosas de sustraer a Camaldoli de la influencia del gobierno mediceo), no debe sorprender, por consiguiente, el interés de los Saboya, archienemigos de los Medici ${ }^{31}$, por dotar a su ducado de una nueva congregación religiosa revistiéndola, ya sea de paso, de caracteres "nacionales" y que, como tal, se propagara fuera de las fronteras del ducado saboyano.

El nuncio apostólico Domenico Ginnasi ${ }^{32}$ no fue ajeno a esta situación, puesto que estaba al tanto de la misma. Antes de instalarse como nuncio en Madrid, había ostentado este mismo cargo en Florencia, lo que le permitió visitar, en calidad de comisario apostólico, a Camaldoli y conocer de cerca la grave crisis abierta en torno a la figura de Alessandro Ceva.

Por otra parte, el duque Carlos Manuel I estaba bien informado de las iniciativas de su cuñado, Felipe III, reflejadas en permitir la fundación de cenobios camaldulenses en suelo español. Por ejemplo, el duque había sido informado de la

29 Zaragoza Pascual, "Primera fundación”, 367-368.

30 Paolo Cozzo, "L'Eremo di Torino e le traversie del suo «venerabile» fondatore", en Gli eremiti camaldolesi di Piemonte: 1601-1801, volume di studi derivato dal convegno svoltosi nel Palazzo comunale di Cherasco il 14 novembre 2015, eds. Gianfranco Armando, Laura Facchin y Diego Lanzardo (Cherasco: Cherasco Cultura, 2017), 1-18.

${ }^{31}$ Franco Angiolini, "Medici e Savoia. Contese per la precedenza e rivalità di rango in età moderna", en L'affermarsi della corte sabauda. Dinastie, poteri, élites in Piemonte e Savoia fra tardo medioevo e prima età moderna, eds. Paola Bianchi y Luisa C. Gentile (Turín: Zamorani, 2006), 435-479.

${ }^{32}$ Giampiero Brunelli, "Ginnasi, Domenico", Diæ̌ionario biografico degli Italiani 55 (2001), en línea: https://www.treccani.it/enciclopedia/domenicoginnasi \%28Dizionario-Biografico $\% 29 /$ (última cons ulta: 20/09/2021). 
aceptación del monarca hispano de las condiciones establecidas por Roma (en particular, la de poner la reciente fundación camaldulense de Sevilla bajo la jurisdicción de Camaldoli, y no bajo la del arzobispo sevillano). Precisamente, la aceptación de las condiciones romanas por parte del joven Felipe III se produjo cuando la negociación entre la corte de Turín, la curia romana y Camaldoli, sobre el grado de autonomía que se debía conceder a la congregación del Piamonte, se había hecho más ardua. Para inducir al duque de Saboya a renunciar a las reivindicaciones autónomas, desde la corte pontificia se le recordó justamente, con respecto a la nueva congregación sevillana, la decisión actuada por el mismo rey de España.

En el fondo, el duque habría tenido que aceptar la forma de proceder de Felipe III, el cual - recordaban desde Roma - "offeriva alla religione camaldulese la fondazione di un eremo a Siviglia senza tante riserve e condizioni, col lasciarlo anzi libero al governo ed alla total dipendenza da quello"33. Pero también el duque, teniendo presente la doble naturaleza (cenobítica y eremítica) de los camaldulenses, cuestionó la manera con la que se estaba actuando en España, donde "quello che dona e vuol fare il re, è monastero" ( $y$, en consecuencia, "è conveniente che sia della medema condizione e natura degli altri monasteri della religione"), y no una ermita. Lo que habría de fundarse en España no era lo mismo que en Piamonte, donde Alessandro Ceva propuso al duque justamente la fundación de una ermita. Al respecto, se afirmaba que "l'eremo è necessario che sia e che si conservi con quella condizione che si conviene all'eremo, il che non seguirebbe quando l'eremo si fondasse o si riducesse alla natura di monastero" 34 .

El resultado de estas largas negociaciones fue favorable a los saboyanos que, si bien reconocieron formalmente la pertenencia de la nueva congregación piamontesa a la familia de Camaldoli, consiguieron de hecho su plena autonomía. Como hemos señalado con anterioridad, la nueva congregación del Piamonte (quizás por interés del propio duque) decidía enviar a Sevilla a tres religiosos, a los que unieron dos religiosos locales. Sin embargo, desde Italia empezó a verse con una cierta perplejidad que se confiara a sujetos, "ch'a pena conoscono le religioni" y "chiamati dal re senza nessuno studio ed opera", la organización de los camaldulenses en territorio hispano ${ }^{35}$.

Esta última expresión ("chiamati dal re"), junto con otras ("los tres padres que el rey dessea" "36 "i padri romiti che il re vuole che vengan per questa fundatione" ${ }^{37}$ ) parecen corroborar la hipótesis de que la elección del soberano, ejercida también sobre los religiosos de la nueva congregación del Piamonte (así como sobre los de Camaldoli,

${ }^{33}$ Es una cita de una carta del embajador saboyano Anastasio Germonio al duque Carlos Manuel I, del 20 de enero de 1601, citado en Cozzo, Un eremita alla corte dei Savoia, 90.

${ }^{34}$ Es una cita de una carta de Alessandro Ceva al duque Carlos Manuel I, del 16 de marzo 1601. Cozzo, Un eremita alla corte dei Savoia, 90.

${ }^{35}$ Es una cita de la carta enviada a Alessandro Ceva por el superior del monasterio camaldulense milanés de San Vito al Carrobbio, el 3 de junio de 1601. Cozzo, Un eremita alla corte dei Savoia, 90.

${ }^{36}$ Es una cita de la carta de Baltasar de Blum al nuncio apostólico en España, 27 de noviembre de 1600, citado en Zaragoza Pascual, "Primera fundación", 382.

${ }^{37}$ Es una cita de la carta de fr. Rodolfo de Valencia al nuncio apostólico en España, 27 de noviembre de 1600, citado en Zaragoza Pascual, "Primera fundación", 382. 
congregación a la que el nuevo asentamiento sevillano había sido sometido), era el resultado de un cálculo político. Tal como fue para otros eclesiásticos "al servizio del re tra Spagna e Italia"38, el recurso de los religiosos camaldulenses podría entonces ser interpretado a la luz de las relaciones políticas y diplomáticas entre Felipe III y el duque Carlos Manuel I, inscribiéndose así en el marco de las relaciones entre las cortes de Madrid y de Turín.

\section{DESTINOS DISTINTOS}

Sin embargo, los destinos de la experiencia camaldulesa en Saboya y en España fueron muy dispares. Mientras que, en los Estados saboyanos, esta experiencia se desarrolló con amplitud y resultó ser muy floreciente durante dos siglos, hasta llegar a la Restauración, en España, en cambio, desembocó en un proyecto fallido. La fundación de Sevilla, pese a que logró la autorización formal del citado nuncio apostólico, en agosto de 1602 todavía no había echado a andar ${ }^{39}$. Este retraso se debió, no sólo a la muerte de dos monjes (probablemente a causa de la "peste atlántica" que golpeó duramente a Sevilla entre 1599 y 160140), sino, sobre todo, porque la protección y beneficencia de Baltasar de Brum fue disminuyendo progresivamente, no sin que ello provocara un "estremo disgusto" en la curia romana, donde era bien sabido que "due grandissimi principi, come è Sua Santità e Sua Maestà, hanno tenuta la mano con l'autorittà loro acciò questo negotio havesse il suo compimento" ${ }^{41}$. Es interesante notar que la noticia de la disolución del proyecto camaldulense de Sevilla no procede de los Annales Camaldulenses -la "memoria oficial" de la Congregación prefirieron "omitir" este asunto -, relacionándose el fracaso del asentamiento sevillano con la muerte del citado Brum. Pero sabemos que, en realidad, este, que hizo su testamento el día 2 de octubre de1604², fallecía años después.

En realidad, Baltasar de Brum, después de abandonar los camaldulenses, en 1603 había ofrecido su ayuda a otra familia religiosa, preferida a la de los monjes italianos: los dominicos. Estos, aunque tomaron posesión de los terrenos en principio destinados a los camalduleses, no permanecieron mucho tiempo en Sevilla ${ }^{43}$. Finalmente, reducidos a un número insignificante y sin recibir suficientes apoyos (entre ellos, los de Brum que se había alejado de los monjes y "mostrava poca voglia di

${ }^{38}$ Elisa Novi Chavarria, ed., "Ecclesiastici a servizio del Re tra Italia e Spagna (secc. XVI-XVII)", numero monográfico de Dimensioni e problemi della ricerca storica 2 (2015).

${ }^{39}$ Zaragoza Pascual, "Primera fundación", 368.

${ }^{40}$ Kristy Wilson Bowers, Plague and Public Health in Early Modern Seville (Rochester: Rochester University Press, 2013), 93-97.

${ }^{41}$ Es una cita de la carta del cardenal protector de la Orden camaldulense, Tolomeo Gallio, al cardenal Fernando Niño de Guevara, arzobispo de Sevilla, citado en Zaragoza Pascual, "Primera Fundación", 391.

42 El testamento, redacto por "Juan García Castellar, escribano público de Sevilla”, es publicado en Miura Andrades, "Baltasar de Brum, albacea testamentario de Arias Montano", 465-475.

43 Poco después la erección canónica del convento dominico (1623) "se iniciaron las conversaciones para trasladarse a Triana, en la ermita de Nuestra Señora de la Candelaria". Miura Andrades, "Baltasar de Brum, albacea testamentario de Arias Montano", 459. 
osservar le promesse"), los camaldulenses abandonaban Sevilla y optaban por retornar a Italia. A este regreso de los camaldulenses a la península donde su Orden había nacido, no quiso (o, quizás, no pudo) oponerse la Monarquía hispana. Desde los tiempos de Felipe II, parecía existir una total frialdad (cuando no, una abierta hostilidad), por parte del resto de instituciones eclesiásticas -y también civiles - de la Monarquía, hacia esta nueva experiencia religiosa, percibida como algo inútil y excesivamente teñida de connotaciones "italianizantes". Es algo no demasiado diferente de lo que habría pasado, en las décadas siguientes, con otra congregación "italiana" (y concretamente "romana"), la de los oratorios de san Filippo Neri". Se trata de una percepción "identitaria" (a nivel de nationes y de marco político de las familias religiosas ${ }^{45}$ ) a la que no fue ajena tampoco la nueva congregación camaldulense del Piamonte, que había nacido con la intención explícita de "independizar" las ermitas del ducado de Saboya del control de Camaldoli y, por tanto, de Florencia.

Los planteamientos aquí esbozados en el asunto del intento de irradiación de los camaldulenses en España confirman, para lo concerniente a la historia del monaquismo de la Edad Moderna, la simbiosis que se produjo entre las sensibilidades religiosas y las dinámicas político-diplomáticas: una simbiosis que encuentra en el complejo mundo de las cortes su natural ( $y$, tal vez, mas adecuado) punto de observación.

${ }^{44}$ Filippo Neri, promotor de la idea que "la reforma y el triunfo de Roma sobre la Monarquía estaba en la espiritualidad radical y no en la armas", fue inspirador "de un comportamiento evangélico modélico y de espiritualidad radical pacífica" en el marco de un catolicismo que "no se basaba en la fuerza militar ni en la ayuda de ningún monarca para conseguir su expansión por el mundo": José Martínez Millán, "El cambio de modelo de santidad durante la segunda mitad del siglo XVII", en Subir a los altares: modelos de santidad en la Monarquía Hispánica (siglos XVI-XVIII), eds. Inmaculada Arias de Saavedra, Ester Jiménez Pablo, Miguel Luis López-Guadalupe Muñoz (Granada: Universidad de Granada, 2018), 102-103.

${ }^{45}$ Massimo Carlo Giannini, ed., Papacy, Religious Orders in the Sixteenth and Seventeenth Centuries (Roma: Viella, 2013). 


\section{REFERENCIAS BIBLIOGRÁFICAS}

Angiolini, Franco, "Medici e Savoia. Contese per la precedenza e rivalità di rango in età moderna", en L'affermarsi della corte sabauda. Dinastie, poteri, élites in Piemonte e Savoia fra tardo medioevo e prima età moderna, eds. Paola Bianchi y Luisa C. Gentile (Turín: Zamorani, 2006), 435-479.

Armando, Gianfranco; Beltramo, Silvia; Cozzo, Paolo y Cuneo, Cristina, I cistercensi foglianti in Piemonte fra corte e chiostro (secoli XVI-XIX) (Roma: Viella, 2020).

Armando, Gianfranco; Facchin, Laura y Lanzardo, Diego, eds., Gli eremiti camaldolesi di Piemonte: 1601-1801, volume di studi derivato dal convegno svoltosi nel Palaz:o comunale di Cherasco il 14 novembre 2015 (Cherasco: Cherasco Cultura, 2017).

Bireley, Robert, The Jesuits and the Thirty Years War: Kings, Courts and Confessors (Cambridge: Cambridge University Press, 2003).

Bolgiani, Franco; Gauna, Gian Franco; Gobbo, Antonio y Goi, Giuseppe, eds, Oratorio e laboratorio: l'intuizione di san Filippo Neri e la figura di Sebastiano V alfré (Bolonia: Il Mulino, 2008).

Bonora, Elena, I conflitti della controriforma. Santità e obbedienza nell'esperienza religiosa dei primi barnabiti (Florencia: Le Lettere, 1998).

Brunelli, Giampiero, "Ginnasi, Domenico”, Diæionario biografico degli Italiani, vol. 55 (2001), en línea: https://www.treccani.it/enciclopedia/domenicoginnasi \%2 8Dizionario-Biografico\%29/(última consulta: 20/09/2021).

Caby, Cecile y Licciardello, Pierluigi, eds., Camaldoli e l'Ordine camaldolese dalle origini alla fine del XV secolo: Atti del I Convegno internazionale di Studi in occasione del millenario di Camaldoli (1012-2012), Monastero di Camaldoli, 31 maggio-2 gingno 2012 (Cesena: Centro storico benedettino italiano, 2014).

Cantarella, Glauco Maria, I castelli della preghiera. Il monachesimo nel pieno medioevo (secoli XXII) (Roma: Carocci, 2020).

Castañiza, Juan, Historia de S. Romualdo padre y fundador de la orden Camaldulense, que es una idea y forma perfecta de la vida solitaria perfecta (Madrid: por el licenciado Castro, 1597).

Caviró Martínez, Balbina, "Los Grecos de don Pedro Laso de la Vega", Goya. Revista de arte 184 (1985): 216-226. 
Chinchilla, Oswaldo, "Pinturas emblemáticas y fiestas teatrales en la época colonial", Conferencias del Museo Pupul Vuh (Museo Pupul Vuh, 2007) https://popolvuh.u fm.edu/wpcontent/uploads/2019/07/Chinchilla2006.pdf, 7.

Cozzo, Paolo, "Bosques y patrimonio forestal en la cultura monástica entre la Edad Media y Edad Moderna: el caso de la Congregación camaldulense", Manuscits (en prensa).

—, "L'Eremo di Torino e le traversie del suo «venerabile» fondatore", en Gli eremiti camaldolesi di Piemonte: 1601-1801 (Volume di studi derivato dal convegno svoltosi nel Palazzo comunale di Cherasco il 14 novembre 2015), eds. Gianfranco Armando, Laura Facchin y Diego Lanzardo (Cherasco: Cherasco Cultura, 2017), 1-18.

—, "La presenza camaldolese in Piemonte", en L'Ordine camaldolese in età moderna e contemporanea, secoli XVI-XX (Atti del II Convegno di studi in occasione del millenario di Camaldoli 1012-2012), eds. Giuseppe M. Croce y Ugo A. Fossa (Cesena: Badia di Santa Maria del Monte, 2015), 207-231.

—, "Le clergé de cour entre service spirituel et fonction politique", en L'État, la cour et la ville. Le duché de Savoie au temps de Christine de France (1619-1663), ed. Giuliano Ferretti (Paris: Classiques Garnier, 2017), 251-269.

-, Un eremita alla corte dei Savoia. Alessandro Ceva e la nascita della Congregazione Camaldolese di Piemonte (Milán: Franco Angeli, 2018).

Cozzo, Paolo y Meyer, Frédéric, "Deux fois, deux lois, une nation? Géographie ecclésiastique et vie religieuse, XVI ${ }^{\mathrm{e}}-\mathrm{XVIII}{ }^{\mathrm{e}}$ siècles", en Les états de Savoie du duché à l'unité d'Italie, ed. Giuliano Ferretti (Paris: Classiques Garnier, 2019), 385428.

Croce, Giuseppe M., "«Mystique et rebellion»: la Congregazione francese degli eremiti camaldolesi", en L'Ordine camaldolese in età moderna e contemporanea, secoli XVI-XX (Atti del II Convegno di studi in occasione del millenario di Camaldoli 1012-2012), eds. Giuseppe M. Croce y Ugo A. Fossa (Cesena: Badia di Santa Maria del Monte, 2015), 233-346.

Croce, Giuseppe Maria y Fossa Ugo A., eds., L'Ordine camaldolese in età moderna e contemporanea, secoli XVI-XX (Atti del II Convegno di studi in occasione del millenario di Camaldoli 1012-2012) (Cesena: Badia di Santa Maria del Monte, 2015).

D'Alessandro Domenico Antonio, Sant'Andrea Avellino e i teatini nella Napoli del viceregno spagnolo. Arte, religione, società (Nápoles: D’Auria, 2011). 
Del Rio Barredo, María José, "El viaje de los príncipes de Saboya a la Corte de Felipe III (1603-1606)", en L'affermarsi della corte sabauda. Dinastie, poteri, élites in Piemonte e Savoia fra tardo medioevo e prima età moderna, eds. Paola Bianchi y Luisa C. Gentile (Turín: Zamorani, 2006), 407-434.

Dovere, Ugo, “«Occuparsi di Dio solo». Temi e figure di spiritualità camaldolese tra Cinquecento e Settecento", en L'Ordine camaldolese in età moderna e contemporanea, secoli XVI-XX (Atti del II Convegno di studi in occasione del millenario di Camaldoli 1012-2012), eds. Giuseppe M. Croce y Ugo A. Fossa (Cesena: Badia di Santa Maria del Monte, 2015), 399-456.

Ernesto Zaragoza, Pascual, "Juan de Castañiza”, en Diccionario biográfico español, en línea: http://dbe.rah.es/biografias/26804/juan de castaniza (última consulta: 20/0 9/2021).

Fossa, Ugo A., Monaci a Camaldoli. Memorie, percorsi, interpretaztioni (Camaldoli: Edizioni Camaldoli, 2020).

Giannini, Massimo Carlo, Papacy, Religious Orders in the Sixteenth and Seventeenth Centuries (Roma: Viella, 2013).

-, Religione, conflittualità e cultura. Il clero regolare nell'Europa d'Antico Regime (Roma: Bulzoni, 2006).

Greco, Gaetano, "Firenze e i camaldolesi dai Medici ai Lorena", en L'Ordine camaldolese in età moderna e contemporánea, secoli XVI-XX (Atti del II Convegno di studi in occasione del millenario di Camaldoli 1012-2012), eds. Giuseppe M. Croce y Ugo A. Fossa (Cesena: Badia di Santa Maria del Monte, 2015), 17-70.

Historia della vita di s. Romualdo. Padre, e fondatore dell'ordine camaldolese, ch'è una idea, e forma perfetta della vita solitaria. Composta dal r.p.d. Gio. da Castagnizza ... Trasportata dalla lingua spagnola nella italiana da $d$. Timotheo da Bagno (Venecia: por Domenico Imberti, 1605).

Hortal Muñoz, José Eloy; Labrador Arroyo, Félix; Bravo Lozano, Jesús y Espíldora García, África, eds., La configuración de la imagen de la Monarquía Católica. El ceremonial de la Capilla Real de Manuel Ribeiro (Madrid: Iberoamericana Vervuert, 2020).

Martínez Hernández, Santiago, "Discreto, artífice y erudito: Un retrato abocetado de don Pedro Laso de la Vega, conde de los Arcos, mayordomo de la reina Margarita de Austria y de Felipe IV (1559-1637)", en Las relaciones discretas entre las Monarquias Hispana y Portuguesa: Las Casas de las Reinas (Siglos XV-XIX), eds. 
José Martínez Millán y María Paula Marçal Lourenço (Madrid: Polifemo, 2008), vol. II, 1187-1220.

Martínez Millán, José, "El cambio de modelo de santidad durante la segunda mitad del siglo XVII", en Subir a los altares: modelos de santidad en la Monarquía Hispánica (siglos XVI-XVIII), eds. Inmaculada Arias de Saavedra, Ester Jiménez Pablo y Miguel Luis López-Guadalupe Muñoz (Granada: Universidad de Granada, 2018), 79-108.

Martínez Millán, José; Rivero Rodríguez, Manuel y Versteegen, Gijs, eds., La Corte en Europa: Política y Religión (siglos XV I-XVIII) (Madrid: Polifemo, 2012).

Martínez Peñas, Leandro, El confesor del Rey en Antiguo Régimen (Madrid: Editorial Complutense, 2007).

Miura Andrades, José María, "Baltasar de Brum, albacea testamentario de Arias Montano y heredero de su finca en Campo de Flores", en Anatomía del bumanismo. Benito Aria Montano, 1598-1998, Homenaje al profesor Melquiades Andrés, ed. Luis Gómez Canseco (Huelva: Universidad de Huelva, 1998), 455-475.

Negredo del Cerro, Fernando, Los predicadores de Felipe IV: corte, intrigas y religión en la España del Siglo de Oro (Madrid: Actas, 2006).

Novi Chavarria, Elisa, ed., "Ecclesiastici a servizio del Re tra Italia e Spagna (secc. XVI-XVII)", numero monográfico de Dimensioni e problemi della ricerca storica 2 (2015).

Pierre, Benoist, La bure et le sceptre: la congregation des Feuillants dans l'affirmation del États et des pouvoirs princiers (vers 1560-vers 1660) (Paris: Publications de la Sorbonne, 2006).

—, La monarchie ecclésiale: le clergé de cour en France à l'époque moderne (Seyssel: Champ Vallon 2013).

Raviola, Blythe A., Giovanni Botero. Un profilo fra storia e storiografia (Milán: Bruno Mondadori, 2020).

Raviola, Blythe A. y Varallo, Franca, eds., L'Infanta. Caterina d'Austria, duchessa di Savoia (1567-1597), (Roma: Carocci, 2013).

Rurale, Flavio, I religiosi a corte: teologia, politica e diplomaria in antico regime: atti del Seminario di studi: Georgetown University a Villa Le Balze, Fiesole, 20 ottobre 1995 (Roma: Bulzoni, 1998). 
Valladares Ramírez, Rafael, La Iglesia en Palacio. Los eclesiásticos en las cortes hispánicas (siglos XVI-XVII) (Roma: Viella, 2019).

Vanni, Andrea, "Fare diligente inquisitione": Gian Pietro Carafa e le origini dei Chierici regolari teatini (Roma: Viella, 2010).

Wilson Bowers, Kristy, Plague and Public Health in Early Modern Seville (Rochester: Rochester University Press, 2013).

Zaragoza Pascual, Ernesto, "Primera fundación de la Orden Camaldulense en España (1597-1602)", Studia monastica 28/2 (1986): 359-391.

Recibido: 7 de julio de 2021 Aceptado: 3 de octubre de 2021 